\title{
Evaluating alternative methods of asset pricing based on the overall magnitude of pricing errors
}

\author{
Qi Shi, ${ }^{\mathrm{a} *}$ Bin $\mathrm{li}^{\mathrm{b}}$ \\ ${ }^{a}$ Department of Finance, Zhe Jiang University of Finance and Economic \\ ${ }^{b}$ Griffith Business School, Griffith University, Australia
}

\begin{abstract}
We are the first pioneers who evaluate the overall fitness of the two-pass Fama-MacBeth regression and the generalized method of moments (GMM) by comparing the $R^{2}$ or mean absolute pricing error (MAE), using a Monte Carlo simulation of different models and portfolios for hundreds of trials and, in particular, focusing on the case that the expected return is always a gross return in both methods. Our findings reveal an innovative finding that both methodologies achieve approximate overall magnitudes of pricing errors.
\end{abstract}

Keywords: Fama-MacBeth regression; GMM; pricing errors; Monte Carlo simulation;

JEL Classifications: G12

*Corresponding author: Qi Shi, qishi@zufe.edu.cn. Department of Finance, Zhe Jiang University of Finance and Economic, Zhe Jiang Province, China. 


\section{Introduction}

As the Hansen (1982) generalized method of moments (GMM) methodology and the Fama-MacBeth (FM) (1973) two-pass regression are not identical and not directly comparable, researchers use both methodologies to circumvent any estimation problems associated with either one of these two methodologies ${ }^{1}$. In addition, the previous literature mostly attempts to compare the efficiency of estimation methods and the size or power of the specification tests of the two methodologies (Jagannathan and Wang (2002) and Lozano and Rubio (2011)).

Our study extends this strand of research and fills an important gap by comparing the magnitude of pricing errors between these two methodologies using gross return rather than excess return in their FM regression equations or GMM moments. We do not particularly concern the case of excess return in the comparison of two methods, since Balvers and Huang (2007, footnote 8 ) point out that the risk premiums using one-stage GMM are very close to those obtained by FM method ${ }^{2}$ in that circumstance. Then, the contribution of our study is a Monto Carlo simulation test on a null hypothesis: the overall magnitudes of pricing errors between these two methodologies are approximate, once comparing these two methodologies on the same scales fairly. Our Monto Carlo simulation particularly reveals that the overall magnitude of the pricing errors of the FM OLS or GLS method and those of first-stage GMM or HJ-GMM ${ }^{3}$ approach is numeridally approximate respectively.

\footnotetext{
${ }^{1}$ Cochrane (2005) states that "the GMM/discount factor, time-series, and cross-sectional regression procedures and distribution theory are similar, but not identical."

${ }^{2}$ Previous empirical studies display approximate estimation of $\mathrm{R}^{2}$ using these two methodologies, such as Maio and Santa-Clara (2012).

${ }^{3}$ HJ-GMM refers to a specific GMM methodology that uses the Hansen and Jagannathan (1997) weighting matrix. Meanwhile, the first-stage GMM refers to a GMM methodology that uses an identity weighting matrix.
} 


\subsection{A brief description of the two alternative methods}

We are the first pioneers who focus on the case where the zero-beta rate is incorporated in the FM regression and the gross return is incorporated in the GMM moments. Again, we note (emphasis) that these two formulations are not directly comparable ${ }^{4}$.

The FM regression is employed in a large body of the asset pricing literature (e.g., Petkova (2006) and Kan et al. (2013)). In the framework of asset pricing, we obtain alternative estimates using Hansen's GMM methodology:

$$
E\left(m r_{i}\right)=1
$$

where $m$ denotes the pricing kernel, $r_{i}$ denotes the gross return of $i$ th portfolio. Cochrane $(2005)^{5}$ presents a transform map between the two-pass FM method and GMM procedure:

$$
\left(E\left(m r_{i}\right)-1\right) / E(m)=E\left(r_{i}\right)-\left(r^{0}-\beta^{\prime} \lambda\right)
$$

where $E\left(m r_{i}\right)-1$ refers to the pricing error $\alpha_{i}$ derived by GMM, $E\left(r_{i}\right)-\left(r^{0}-\beta^{\prime} \lambda\right)$ alternatively represents the pricing errors of the FM regression, and $r^{0}$ or $1 / E(m)$ denotes the zero-beta rate. This transform map implies that: once the former of equation is scaled down by the amount of the zero-beta rate, the overall pricing errors of two methods achieve a fair comparison.

\subsection{Measures of the models' overall goodness of fit}

Following in the spirit of previous studies (Hsu and Huang (2010), Maio and Santa-Clara (2012)), we use the mean absolute pricing error (MAE) and $\mathrm{R}^{2}$ as our goodness-of-fit measure. Furthermore, Maio and Santa-Clara (2012) show that the MAE and $\mathrm{R}^{2}$ statistics are robust and more powerful than some specification tests. The MAE is calculated as follows:

\footnotetext{
${ }^{4}$ In particular, these two formulations suggest different ways of estimating the covariance price of risk; the GMM approach is equivalent to computes a cross-sectional regression of the average price (i.e., a vector of ones) on a second-moments matrix of payoffs and factors, while the FM approach estimates a cross-sectional regression of the average gross return on the covariance matrix of (demeaned) payoffs and factors.

${ }^{5}$ Please see Cochrane (2005) for details.
} 


$$
M A E=\frac{1}{N} \sum_{i=1}^{N}\left|\alpha_{i}\right|,
$$

where $\alpha_{i}$ represents the pricing errors of $N$ moments in GMM. The $\mathrm{R}^{2}$ of the first-stage GMM regression is evaluated as follows:

$$
R_{O L S}^{2}=1-\frac{\overline{\hat{\alpha}}^{\prime} \overline{\hat{\alpha}}}{\bar{R}^{\prime} \bar{R}},
$$

where $\overline{\hat{\alpha}}$ denotes the vector of demeaned pricing errors and $\bar{R}$ denotes the vector of the demeaned gross return.

\section{Monte Carlo simulation design}

Following the light of Ahn and Gadarowski (2004), we simulate the data setup in two simulation settings. In the first setting, we assume that all factors are independent and identically distributed (i.i.d.), while in the second setting, we allow cross-sectional correlations among the factors and asset returns. In the first setting, we simulate not only a simple three-factor model (hereafter called a simple model), but also 25 assets and 100 assets in time-series observations from 600 and 1000 periods. In the second setting, we simulate Petkova's (2006) intertemporal capital asset pricing model (ICAPM) and Fama and French's (2015) five-factor model and Fama-French 25 size and value portfolios (SBM25), 100 size and value portfolios (SBM100), 25 size and long-term reversal portfolios, 100 size and investment portfolios, and Fama-French industry portfolios, where the source of original data follows original literatures (Maio and Santa-Clara (2012), Fama and French (2015), Fama and French (2016), Lioui and Maio (2014)). The period of sample is between July 1963 and September 2014.

We generate 1000 unique simple data sets (1000 trials) and 1000 different calibrated data sets (1000 trials). We calculate the frequency at which the absolute value of the difference of the 
$\mathrm{R}^{2}$ or the MAE is less than 0.01 or $0.01 \%$, respectively; additionally, we refer to the frequency as a P-value in the Monte Carlo simulation ${ }^{6}$. We choose the criteria of 0.01 and $0.01 \%$ because they represent the efficient and accurate positive (statistical) values of the $\mathrm{R}^{2}$ and the MAE to two decimal places (Maio and Santa-Clara (2012)).

\section{Simulation results}

\section{[Insert Table 1 here]}

Table 1 shows the overall goodness of fit of the two-pass OLS/GLS regression and the first stage of the GMM/HJ-GMM approach. Panel A of Table 1 reports the/results of simulations where each trial uses Ahn and Gadarowski's (2004) simple three-factor model to price 25 and 100 portfolios; Panels B and C of Table 1 provide the results of other simulations, where each simulation trial uses a calibrated model (i.e., Petkova's (2006) ICAPM or Fama and French's (2015) five-factor model) to price the calibrated SBM25 (relaxed via 12 industrial portfolios) and SBM100 (relaxed via 25 industrial portfolio) ${ }^{7}$; we set the time periods as 600 and 1000 observations $^{8}$, respectively. Clearly, all the reported P-values are significantly higher than 0.99 , suggesting that the OLS FM regression and the first-stage GMM regression achieve the same $\mathrm{R}^{2}$; meanwhile, the GLS FM regression and the HJ-GMM approach numerically achieve the same MAE.

As Lewellen et al. (2010) provide some criticisms of the validity of cross-sectional asset pricing tests that rely exclusively on size and book-to-market sorted portfolios, we simulate other testable portfolios (following Lioui and Maio (2014) and Fama and French (2016)) as a

\footnotetext{
${ }^{6}$ To test the hypothesis that the FM two-pass regression and the GMM methodology produce identical pricing errors one can simply compare the reported P-values in which the acceptable level of significance is $0.95(1-0.05=0.95)$, where 0.05 is the level of significance in statistics for a reject rate.

${ }^{7}$ We follow Lewellen et al.'s (2010) suggestion: to relax the strong factor structure, we add 12 industrial portfolios to SBM25 or add 30 industrial portfolios to SBM100.

${ }^{8} 600$ observations are commonly thought to represent a typical finite sample and 1000 observations a relative large sample.
} 
robustness check in Table 2. This section reports the overall fitness of the two-pass OLS/GLS regression and the first stage of the GMM/HJ-GMM approach in a Monte Carlo simulation with 1000 trials, where each simulation trial uses the calibrated model (Petkova's (2006) model or Fama and French's (2015) five-factor model) to price the calibrated 25 size and long-term reversal portfolios and 100 size and investment portfolios; the time period remains at 600 and 1000 observations, respectively. Notably, all the reported P-values are approximately equivalent to 1.00; therefore, we accept our null hypothesis that the OLS FM regression and first-stage GMM regression numerically achieve the same $\mathrm{R}^{2}$ and that the GLS FM regression and HJGMM regression numerically achieve the same MAE.

[Insert Table 2 here]

\section{Concluding remarks}

After adjusting the pricing errors for a scaling factor, our evidence from the Monte Carlo simulation illustrates that the overall magnitude of the pricing errors of GMM (the gross return case) and FM regression (the zero-beta case) is approximate. 


\section{References}

Ahn, S. C., \&Gadarowski, C. (2004). Small sample properties of the GMM specification test based on the Hansen-Jagannathan distance. Journal of Empirical Finance, 11(1), 109132.

Cochrane, J. H.2005. Asset Pricing. Princeton: Princeton University Press.

Fama, E. F., \&MacBeth, J. D. (1973). Risk, return, and equilibrium: Empirical tests. Journal of political economy, 81(3), 607-636.

Fama, E. F., \& French, K. R. (2015). A five-factor asset pricing model. Journal of Financial Economics, 116(1), 1-22.

Fama, E. F., \& French, K. R. (2016). Dissecting anomalies with a five-factor model. The Review of Financial Studies, 29(1), 69-103.

Hansen, L. P. (1982). Large sample properties of generalized method of moments estimators. Econometrica: Journal of the Econometric Society, 1029-1054.

Hansen, L. P., \& Jagannathan, R. (1997). Assessing specification errors in stochastic discount factor models. The Journal of Finance, 52(2), 557-590.

Jagannathan, R., \& Wang, Z. (2002). Empirical Evaluation of Asset- Pricing Models: A Comparison of the SDF and Beta Methods. The Journal of Finance, 57(5), 2337-2367.

Lewellen, J., Nagel, S., \&Shanken, J. (2010). A skeptical appraisal of asset pricing tests. Journal of Financial economics, 96(2), 175-194.

Lioui, A., \&Maio, P. (2014). Interest rate risk and the cross section of stock returns. Journal of Financial and Quantitative Analysis, $49(2)$, 483-511.

Lozano, M., \& Rubio, G. (2011). Evaluating alternative methods for testing asset pricing models with historical data. Journal of Empirical Finance, 18(1), 136-146.

Maio, P., \& Santa-Clara, P. (2012). Multifactor models and their consistency with the ICAPM. Journal of Financial Economics, 106(3), 586-613.

Petkova, R. (2006). Do the Fama-French factors proxy for innovations in predictive variables? The Journal of Finance, 61(2), 581-612 
Table 1 Simulation results for a combination of SBM portfolios and industrial portfolios

\begin{tabular}{|c|c|c|c|c|}
\hline \multirow{2}{*}{$\begin{array}{l}\text { Time-series } \\
\text { observation }\end{array}$} & \multicolumn{2}{|c|}{25 assets } & \multicolumn{2}{|c|}{100 assets } \\
\hline & OLS $R^{2}$ & GLS MAE & OLS $R^{2}$ & GLS MAE \\
\hline \multicolumn{5}{|c|}{ Panel A $\quad$ Model 1: A simple three-factor model } \\
\hline 600 & 0.973 & 0.027 & 0.972 & 0.029 \\
\hline P-value & 1.000 & 1.000 & 1.000 & 1.000 \\
\hline 1000 & 0.984 & 0.021 & 0.983 & 0.023 \\
\hline P-value & 1.000 & 1.000 & 1.000 & 1.000 \\
\hline \multicolumn{5}{|c|}{ Panel B $\quad$ Model 2: Petkova ICAPM } \\
\hline 600 & 0.516 & 0.114 & 0.483 & \\
\hline P-value & 1.000 & 1.000 & 1.000 & \\
\hline 1000 & 0.622 & 0.094 & 0.580 & \\
\hline P-value & 1.000 & 1.000 & 1.000 & \\
\hline \multicolumn{5}{|c|}{ Panel C Model 3: Fama-French five-factor model } \\
\hline 600 & 0.828 & 0.058 & 0.823 & 0.203 \\
\hline P-value & 1.000 & 1.000 & 1.000 & 1.000 \\
\hline 1000 & 0.887 & 0.045 & 0.880 & 0.164 \\
\hline P-value & 1.000 & 1.000 & 1.000 & 1.000 \\
\hline
\end{tabular}

We use Ahn and Gadarowski's (2004) simple data-generating process to simulate a simple three-factor model, with 25 portfolios and 100 portfolios for 600 and 1000 trials, respectively, and report the results in Panel A. In Panels B and $\mathrm{C}$, we report the results for a simulation where the calibrated data-generating process is used to simulate Petkova's (2006) ICAPM and the Fama-French (2015) five-factor model, with SBM 25 (relaxed via 12 industrial portfolios) and SBM 100 portfolios (relaxed via 30 industrial portfolios) for 600 and 1000 trials, respectively. We use the FM method and the GMM approach to evaluate the performance of models using simulated data for hundreds of trials. The GLS MAE presents the mean value of the average absolute pricing errors (in \%) in the FM GLS method or the approximate mean value of the average absolute pricing errors for HJ-GMM approach. OLS $R^{2}$ denotes the mean value of the OLS cross-sectional $R^{2} s$ in the FM OLS method or the approximate mean value for $R^{2} s$ of first stage GMM approach. P-value of $R^{2}$ refers to the probability that each absolute value of the difference of the $R^{2}$ is less than 0,01 in hundreds of trials, and P-value of MAE denotes the probability that each absolute value of the difference of the MAE is less than $0.01 \%$ in hundreds of trials. 
Table 2 Simulation results for other testing portfolios

\begin{tabular}{lcccc}
\hline \multirow{2}{*}{$\begin{array}{l}\text { Time-series } \\
\text { observation }\end{array}$} & \multicolumn{3}{c}{25 assets } & \multicolumn{3}{c}{ assets } \\
\cline { 2 - 5 } & OLS $R^{2}$ & GLS MAE & OLS $R^{2}$ & GLS MAE \\
\hline Panel A & Model 2: Petkova ICAPM & & & \\
\hline 600 & 0.600 & 0.097 & 0.781 & 0.241 \\
P-value & 1.000 & 1.000 & 1.000 & 1.000 \\
1000 & 0.708 & 0.078 & 0.854 & 0.207 \\
P-value & 1.000 & 1.000 & 1.000 & 1.000 \\
\hline & Panel B & Model 3: Fama-French five-factor model & \\
\hline 600 & 0.842 & 0.057 & 0.899 & 0.166 \\
P-value & 1.000 & 1.000 & 1.000 & 1.000 \\
1000 & 0.896 & 0.045 & 0.940 & 0.133 \\
P-value & 1.000 & 1.000 & 1.000 & 1.000 \\
\hline
\end{tabular}

In Panels $\mathrm{A}$ and $\mathrm{B}$, we report the results for a simulation where the calibrated data-generating process is used to simulate Petkova's (2006) ICAPM and the Fama-French (2015) five-factor model, with 25 size and long-term reversal portfolios and 100 size and investment portfolios for 600 and 1000 trials, respectively. We use the FM method and the GMM approach to evaluate the performance of the models using simulated data for hundreds of trials. See the notes of Table 1 for other definitions. 